tion, it now has all real amplitudes. The $B$ values, amplitudes, and direction cosines of the principal axes are (in the order $B$, amplitude, $\varphi, \psi, \omega)$ :

$$
\begin{aligned}
& 3.42 \AA^{2}, 0 \cdot 208 \AA,-0 \cdot 316,-0.685,0.706 ; \\
& 2 \cdot 62 \AA^{2}, 0 \cdot 182 \AA, 0.945,-0 \cdot 165,0.094 ; \\
& 1.55 \AA^{2}, 0 \cdot 140 \AA,-0.085,0.711,0.702
\end{aligned}
$$

\section{References}

Burns, J. H. \& Waser, J. (1957). J. Amer. Chem. Soc. 79, 859.
Camerman, N. \& Trotrer, J. (1963). Acta Cryst. 16, 922. EMERSON, K. \& Britton, D. (1963). Acta Cryst. 16, 113. Emerson, K. \& Britton, D. (1964). Acta Cryst. 17, 1134. GrichKiEWITCH-TROHIMOWSKI, M. E., MateyaK, L. \& ZABlorski, N. N. (1927). Bull. Soc. Chim. France, 41, 1323.

Hamilton, W. C. (1965). Acta Cryst. 18, 502.

Hedberg, K., Hughes, E. W. \& Waser, J. (1961). Acta Cryst. 14, 369.

HiRshFeLD, F. (1964). Israel J. Chem. $2,87$.

Long, L. H., Emeleus, H. J. \& Briscoe, H. V. A. (1946). J. Chem. Soc. p. 1123.

Maier, L. (1963). Helv. Chim. Acta, 46, 2667.

Acta Cryst. (1966). 20, 783

\title{
The Crystal Structure of Imidazole at $-150^{\circ} \mathrm{C}$
}

\author{
By SAgrario Martinez-CARRERA* \\ Laboratorium voor Kristallografie, University of Amsterdam, The Netherlands
}

(Received 2 September 1965)

\begin{abstract}
Imidazole, $\mathrm{C}_{3} \mathrm{~N}_{2} \mathrm{H}_{4}$, crystallizes in the space group $P 2_{1} / c$. There are four molecules in the cell, the dimensions of which are, at $-150^{\circ} \mathrm{C}: a=7.582, b=5.371, c=9 \cdot 790 \AA ; \beta=61^{\circ} 1^{\prime}$. The five-membered ring is planar. All bond lengths in the ring indicate considerable double-bond character. $\mathrm{NH} \cdots \mathrm{N}$ bonds with the exceptionally short length of $2.86 \AA$ form chains of molecules along the $c$ axis.
\end{abstract}

\section{Introduction}

The study of the crystal structure of imidazole was started in the Instituto de Fisica Alonso de Santa Cruz (García Blanco \& Martinez-Carrera, 1958). A tentative projection of the structure was given, but the X-ray data, taken at room temperature, were insufficient for refinement. Low-temperature data were collected and the structure solved in 1958/59 in this laboratory, while the author held a Netherlands-Spain exchange fellowship. The structure was further refined in 1963 in the Laboratory of Crystallography of the University of Pittsburgh by courtesy of Prof. G. A. Jeffrey. In the meantime Will (1963), apparently unaware of our former work, published a rough trial structure of imidazole at room temperature. Since our experimental low-temperature data are far more complete, they permit an accurate determination of the molecular geometry and of the packing in the crystal.

\section{Experimental}

Imidazole, with molecular formula

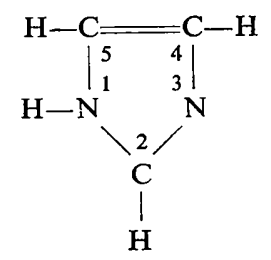

* Present address: Instituto de Quimica Fisica 'Rocasolano', Serrano 119, Madrid 6, Spain. crystallizes in the monoclinic system, class $2 / \mathrm{m}$. Single crystals elongated along the $b$ axis were obtained from ethanol solution. The cell constants were determined by least squares, both at room temperature and at $-150^{\circ} \mathrm{C}$, from $\sin ^{2} \theta$ values of $h k 0$ and $h 0 l$ reflexions. These were obtained from Weissenberg diagrams calibrated with aluminum powder lines. The values found were:

$$
\begin{aligned}
& \begin{array}{ccccccc} 
& a & \sigma_{a} & b & \sigma_{b} & c & \sigma_{c} \\
20^{\circ} \mathrm{C} & 7.732 & 0.002 & 5.453 & 0.002 & 9.766 & 0.002 \AA
\end{array} \\
& \begin{array}{rrrrrrr}
-150^{\circ} \mathrm{C} & 7.582 & 0.002 & 5.371 & 0.002 & 9.790 & 0.003 \AA
\end{array}
\end{aligned}
$$

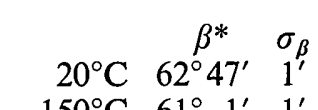

The room temperature values of Will are in somewhat better agreement with those given above than those of García Blanco \& Martinez-Carrera.

By comparison of the films it was clear that the structure is basically the same at $-150^{\circ} \mathrm{C}$ and at room temperature. The space group is $P 2_{1} / c$. With four formula units per unit cell the calculated density is $1.297 \mathrm{~g} . \mathrm{cm}^{-3}$ at $-150^{\circ} \mathrm{C}$.

The crystals were mounted in thin-walled glass capillaries and the X-ray intensities were obtained on a Weissenberg camera. Cooling of the crystal to $-150^{\circ} \mathrm{C}$ was achieved by a gas stream passed through liquid air (Kreuger, 1955).

The X-ray diffraction intensities were recorded by a multiple-film technique with $\mathrm{Cu} K$ radiation. Five

\footnotetext{
* $\beta$ is taken acute, conforming with the original setting of Greenwood (1926).
} 
layers about the $a$ axis and five layers about the $b$ axis were recorded. The intensities were evaluated by photometric measurement; for non-equatorial layers they were corrected for spot length following the Phillips (1956) method. No corrections were made for absorption because the absorption coefficient is very small, $\mu=7.5 \mathrm{~cm}^{-1}$; even for the largest specimen used it is estimated that the corrections would produce variations of less than $5 \%$ in the values of the structure amplitudes.

After reduction to structure factors, the latter were correlated by means of common reflexions on different films. 584 independent $h \mathrm{kl}$ reflexions were observed representing $77 \%$ of the possible number observable.

\section{The determination of the structure}

The structure was essentially solved by interpreting the three-dimensional Patterson synthesis. As the molecule of imidazole has no centre of symmetry, all atoms must be in general positions. The pentagonal imidazole ring was assumed planar, with distances between neighbouring carbon-carbon and carbon-nitrogen atoms taken as $1.4 \AA$ and distances between next nearest neighbours as $2 \cdot 2 \AA$. Initially no distinction was made between nitrogen and carbon atoms, an ambiguity which was not resolved during the interpretation of the Patterson function.

The orientation of the molecules was found by plotting in stereographic projection the values of the Patterson function on the surfaces of spheres around the origin with radii of 1.4 and $2 \cdot 2 \AA$, respectively. The molecular orientation found by García Blanco and Martinez-Carrera (1958) was approximately confirmed. However, by looking for the intermolecular vectors, we found that the location of the molecule with respect to the origin as given in their paper was about $0.5 \AA$ off. The trial structure derived from the Patterson function was first refined in projections along the $a$ and $b$ axes simultaneously. After several cycles all atoms were resolved in both projections and the nitrogen atoms were distinguished by having a slightly larger electron density (Figs. 1 and 2).

Already the first difference synthesis of the $h 0 l$ reflexions showed all four hydrogen atoms(Fig.3). Further refinement was carried out in three-dimensional space by difference Fourier synthesis. The residual $R$ was then 0.141 for all observed reflexions, with the hydrogen contributions included in the calculated structure factors, and an overall isotropic temperature factor $B=$ $1.78 \AA^{2}$. Finally, the structure was refined by five cycles of differential synthesis, with a program written by Shiono (1962) for the IBM 7070 computer. In this refinement, all atomic positions were allowed to vary; however, the shifts for the hydrogen atoms appeared not relevant and therefore were not used. Individual isotropic temperature factors were computed for the carbon and nitrogen atoms; for hydrogen a uniform $B$ of $2 \cdot 14 \AA^{2}$ proved to give the best fit.
The final $R$ for observed reflexions only was $0 \cdot 112$. Structure factors of unobserved reflexions were invariably calculated to be small.

The final calculated and observed structure factors are given in Table 1 . The atomic parameters with their standard deviations are listed in Table 2 . The bond angles and bond lengths are shown in Table 3. The mean e.s.d. in bond lengths in the imidazole ring is $0.005 \AA$ and in bond angles $0 \cdot 3^{\circ}$. The hydrogen bond length $2.86 \AA$ is exceptionally short. In a recent survey of hydrogen bonds (Wallwork, 1962), $\mathrm{NH} \cdots \mathrm{N}$ is given as $3.07 \pm 0.11 \AA ; \mathrm{N}^{+}-\mathrm{H} \cdots \mathrm{N}$ as $2.92 \pm 0.05 \AA$; $\mathrm{N}^{+}-\mathrm{H} \cdots \mathrm{N}^{-}$as $2.93 \pm 0.05 \AA$.

It appears therefore from the length of the hydrogen bond that at least the nitrogen atom N(1) carries a positive formal charge.

Other intermolecular approaches less than $4.0 \AA$ are listed in Table 5. The least-squares plane through the

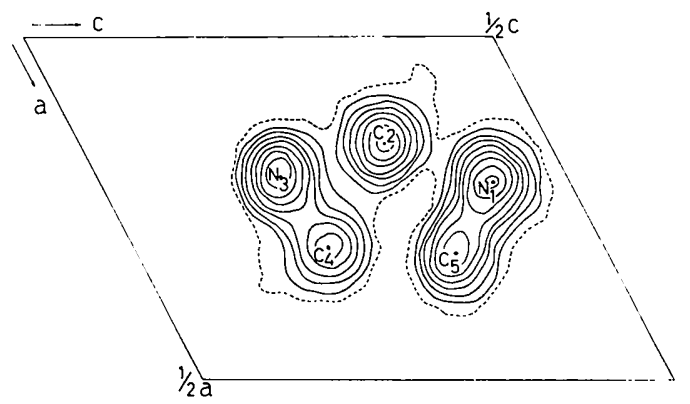

Fig. 1. Imidazole. Electron density projection along [010].

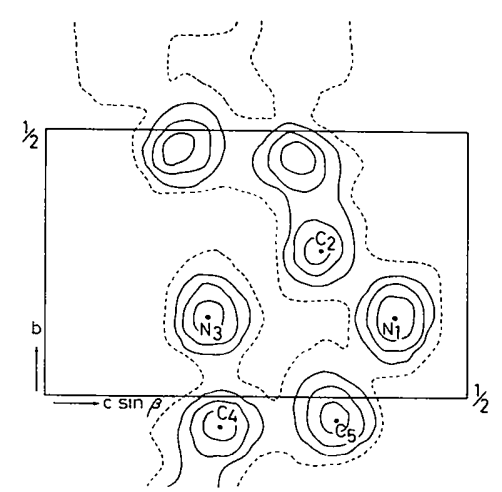

Fig. 2. Imidazole. Electron density projection along [100].

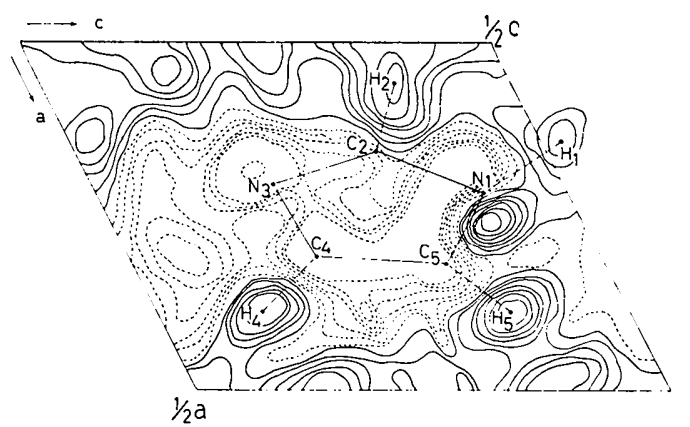

Fig. 3. Imidazole. Difference Fourier synthesis along [010]. 
Table 1. Observed and calculated structure factors

Reflexions marked with an asterisk were unobserved.

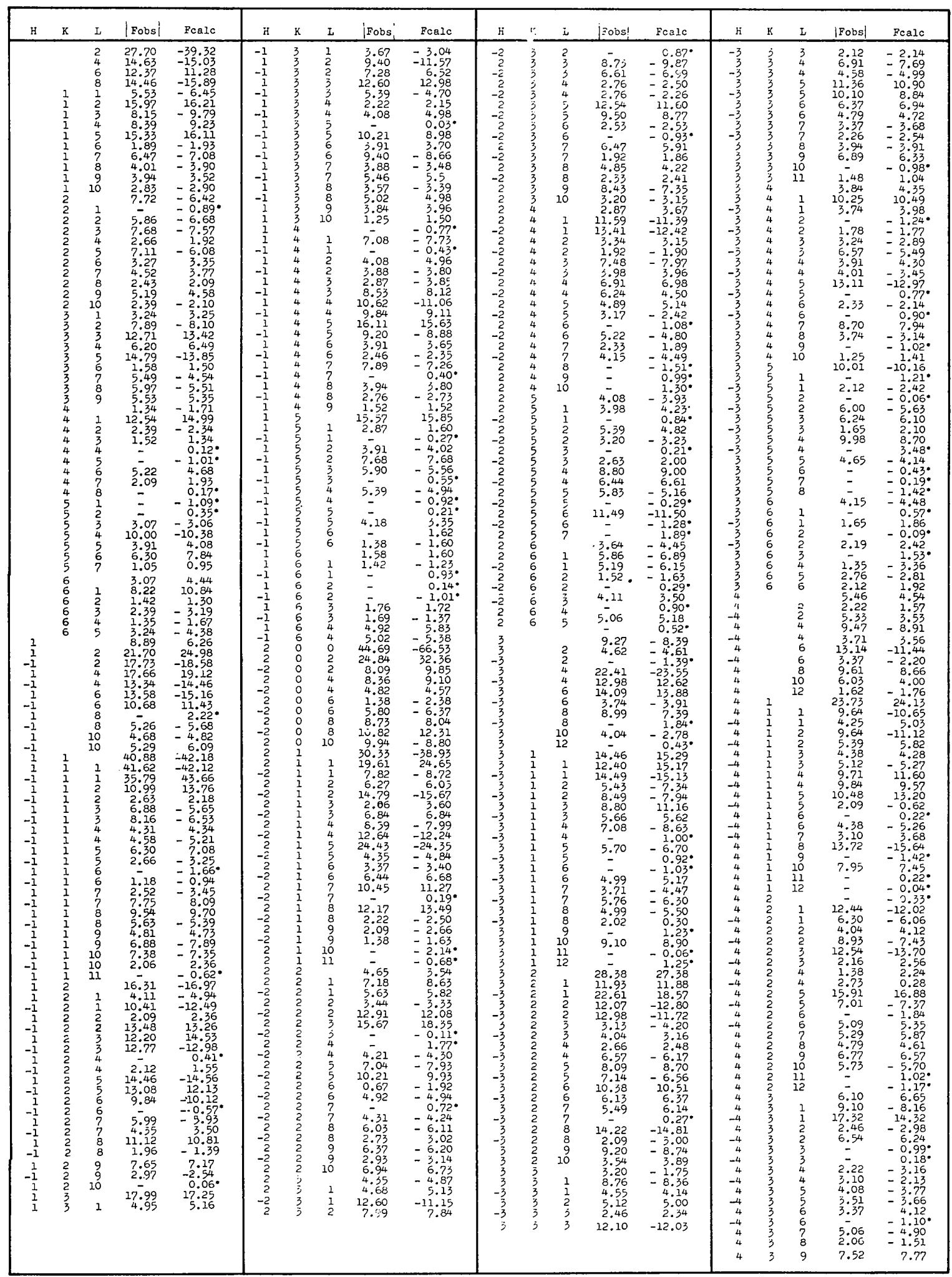


Table 1 (cont.)

\begin{tabular}{|c|c|c|c|c|c|c|c|c|c|c|c|c|c|c|}
\hline H & $K$ & I & Fobs & Feale & н & K & L & Zobs & Foalc & H & $\ddot{K}$ & I & Fobs & $x_{\text {roalc }}$ \\
\hline $\begin{array}{l}4 \\
4\end{array}$ & $\frac{3}{3}$ & $\begin{array}{l}10 \\
11\end{array}$ & 1.48 & & $\begin{array}{l}6 \\
6\end{array}$ & & $\frac{6}{8}$ & $\begin{array}{l}11.05 \\
15.657\end{array}$ & & $?$ & 4 & 8 & $\begin{array}{l}3.67 \\
5.60\end{array}$ & $=\frac{3.06}{3.06}$ \\
\hline $\begin{array}{r}4 \\
4 \\
-4 \\
-4\end{array}$ & $\begin{array}{l}4 \\
4 \\
4\end{array}$ & $\frac{1}{1}$ & $\begin{array}{l}6.57 \\
5.66 \\
6.17\end{array}$ & & $\begin{array}{l}0 \\
6 \\
6 \\
6\end{array}$ & & 12 & $\begin{array}{l}2.40 \\
8.99\end{array}$ & 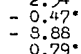 & $\begin{array}{l}8 \\
8 \\
8 \\
8\end{array}$ & & $\begin{array}{l}4 \\
4 \\
6 \\
8\end{array}$ & $\begin{array}{r}3.79 \\
0.787 \\
0.67 \\
1146\end{array}$ & $\begin{array}{r}-9.15 \\
=9.15 \\
=1.45\end{array}$ \\
\hline$\frac{4}{4}$ & $\begin{array}{l}4 \\
4 \\
4\end{array}$ & $\begin{array}{l}2 \\
2 \\
3\end{array}$ & $\begin{array}{l}3.64 \\
8.86\end{array}$ & $\begin{array}{l}0.72 \\
2.97 \\
8.00\end{array}$ & $\begin{array}{l}-6 \\
-6 \\
6\end{array}$ & $\frac{1}{1}$ & $\frac{1}{2}$ & 2.66 & $\begin{aligned}-0.772^{\circ} \\
3 \\
3.02\end{aligned}$ & 8 & 1 & $10^{\circ}$ & $\begin{array}{r}1.40 \\
4.11 \\
1.69\end{array}$ & $\begin{array}{r}1.09 \\
-4.15 \\
2.25 \\
3.24\end{array}$ \\
\hline $\begin{array}{c}-4 \\
-4 \\
-4\end{array}$ & $\begin{array}{l}4 \\
4 \\
4\end{array}$ & & $\begin{array}{l}\text { 2.16.16 } \\
\text { s.6. } \\
3.64\end{array}$ & $\begin{array}{r}-2.29 \\
-8.45 \\
-3.66\end{array}$ & $\begin{array}{l}-6 \\
-6 \\
-6 \\
-6\end{array}$ & 1 & $\begin{array}{l}\frac{2}{3} \\
\vdots \\
j\end{array}$ & $\begin{array}{l}2.55 \\
2.19\end{array}$ & $\begin{aligned}-0.0 .50^{\circ} \\
= \\
2.15 \\
2.53\end{aligned}$ & $\begin{array}{l}8 \\
8 \\
6\end{array}$ & & $\frac{1}{2}$ & $\begin{array}{l}3.000 \\
2.00 \\
1.92\end{array}$ & $\begin{array}{l}3.38 \\
2.76 \\
1.85 \\
1.85\end{array}$ \\
\hline $\begin{array}{r}-4 \\
-4 \\
4\end{array}$ & $\begin{array}{l}4 \\
4 \\
4\end{array}$ & $\begin{array}{l}5 \\
5 \\
6\end{array}$ & $\begin{array}{l}5.59 \\
2.46 \\
6.44 \\
6.44\end{array}$ & $\begin{array}{r}-4.68 \\
-2.59 \\
-6.68\end{array}$ & $\begin{array}{c}6 \\
-6 \\
6\end{array}$ & $\frac{1}{1}$ & $\begin{array}{l}4 \\
4 \\
5\end{array}$ & 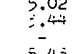 & $\begin{array}{r}-5.399 \\
=4.25 \\
-0.38 \\
5\end{array}$ & $\begin{array}{l}8 \\
8 \\
5 \\
9\end{array}$ & & $\begin{array}{l}4 \\
5 \\
6 \\
7\end{array}$ & $\begin{aligned} 5.20 \\
5.24 \\
4.52\end{aligned}$ & 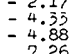 \\
\hline $\begin{array}{l}4 \\
4 \\
4 \\
4\end{array}$ & & $\begin{array}{r}7 \\
8 \\
9 \\
10\end{array}$ & $\begin{array}{l}4.43 \\
4: 11 \\
1: 01 \\
1.01\end{array}$ & $\begin{array}{r}4.98 \\
4.05 \\
-1.43 \\
-1.00\end{array}$ & $\begin{array}{l}0 \\
6 \\
6 \\
6\end{array}$ & 1 & $\begin{array}{r}0 \\
7 \\
8 \\
9 \\
\end{array}$ & $\begin{array}{l}3.45 \\
5.60 \\
5.81 \\
6.34\end{array}$ & $\begin{array}{r}-3.02 \\
-3.14 \\
7.21 \\
6.17\end{array}$ & $\begin{array}{l}3 \\
8 \\
8 \\
8 \\
8\end{array}$ & & $\begin{array}{r}8 \\
9 \\
10 \\
10\end{array}$ & 2.11 & $\begin{array}{r}1.49 \\
-7.49 \\
-2.30 \\
-2.32\end{array}$ \\
\hline $\begin{array}{l}4 \\
4 \\
\end{array}$ & $\begin{array}{l}5 \\
5 \\
5\end{array}$ & $\frac{1}{1}$ & $\begin{array}{l}2.56 \\
2.83 \\
-\quad\end{array}$ & $\begin{array}{r}2 \\
=3.44 \\
= \\
=0.98 \\
0.95\end{array}$ & $\begin{array}{l}6 \\
6 \\
6\end{array}$ & $\frac{1}{4}$ & $\begin{array}{l}10 \\
11 \\
12\end{array}$ & & $\begin{array}{r}-6.92 \\
0.67 \\
1.35\end{array}$ & $\begin{array}{l}8 \\
8 \\
8\end{array}$ & & $\begin{array}{r}11 \\
1\end{array}$ & $\begin{array}{l}2.56 \\
2.50 \\
2.80\end{array}$ & 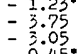 \\
\hline $\begin{array}{r}4 \\
-4 \\
4\end{array}$ & $\begin{array}{l}5 \\
5 \\
5\end{array}$ & $\begin{array}{l}\frac{2}{2} \\
2 \\
3\end{array}$ & $\begin{array}{l}6.24 \\
2.19 \\
3.44\end{array}$ & $\begin{array}{r}-5.46 \\
-4.18 \\
3.61 \\
3.61\end{array}$ & $\begin{array}{c}6 \\
6 \\
-6\end{array}$ & $\begin{array}{l}2 \\
2 \\
2 \\
2\end{array}$ & $\frac{1}{1}$ & $\begin{array}{l}8.36 \\
2.73\end{array}$ & $\begin{array}{l}2.15^{\circ} \\
73.80 \\
3.07\end{array}$ & $\begin{array}{l}8 \\
8 \\
8\end{array}$ & $\begin{array}{l}2 \\
2 \\
\hat{2} \\
\hat{2}\end{array}$ & $\begin{array}{l}2 \\
3 \\
4\end{array}$ & $\begin{array}{l}0.49 \\
2.72\end{array}$ & $\begin{array}{r}0.45^{\circ} \\
-1.5 .58 \\
7.58\end{array}$ \\
\hline $\begin{array}{r}-4 \\
4 \\
4 \\
4\end{array}$ & $\begin{array}{l}5 \\
5 \\
5 \\
5\end{array}$ & $\begin{array}{l}3 \\
3 \\
5 \\
5 \\
6\end{array}$ & $\begin{array}{l}3.50 \\
3.49 \\
6.48\end{array}$ & $\begin{array}{r}0.366^{\circ} \\
-5.395 \\
1.97 \\
7.07\end{array}$ & $\begin{array}{l}6 \\
-6 \\
-6 \\
-6\end{array}$ & $\begin{array}{l}2 \\
2 \\
2 \\
2 \\
2\end{array}$ & $\begin{array}{l}2 \\
2 \\
3 \\
3\end{array}$ & $\begin{array}{c}\bar{z} \\
4.99 \\
2.02\end{array}$ & $\begin{array}{l}=0.45^{\circ} \\
-5.40 \\
-2.40 \\
-2.42\end{array}$ & $\begin{array}{l}8 \\
8 \\
8 \\
8\end{array}$ & $\begin{array}{l}\frac{1}{2} \\
\dot{2} \\
\frac{2}{2} \\
2\end{array}$ & $\begin{array}{l}5 \\
0 \\
7 \\
8\end{array}$ & $\begin{array}{l}5.66 \\
5.56 \\
=\end{array}$ & $\begin{array}{r}6.48 \\
=5.68 \\
-\quad 0.24 * \\
-\quad 0.93^{*}\end{array}$ \\
\hline $\begin{array}{l}4 \\
4 \\
4 \\
4\end{array}$ & & $\begin{array}{l}6 \\
? \\
8\end{array}$ & $\begin{array}{l}6.88 \\
= \\
3.67\end{array}$ & $\begin{array}{r}7.08 \\
-1.03: \\
0.41 \\
4.01 \\
4.01\end{array}$ & $\begin{array}{l}-6 \\
6 \\
-6 \\
6\end{array}$ & $\begin{array}{l}2 \\
2 \\
2 \\
2 \\
2\end{array}$ & $\begin{array}{l}3 \\
4 \\
4 \\
5\end{array}$ & $\begin{array}{r}2.55 \\
2.55 \\
2.39 \\
11.86\end{array}$ & $\begin{array}{r}-7.88 \\
3.20 \\
-13.00\end{array}$ & $\begin{array}{l}8 \\
8 \\
8\end{array}$ & $\begin{array}{l}2 \\
2 \\
2 \\
3 \\
3\end{array}$ & $\begin{array}{r}0 \\
9 \\
10 \\
2\end{array}$ & $\bar{z}$ & $\begin{array}{r}-0.72 \\
1.91 \\
-1.25\end{array}$ \\
\hline & $\begin{array}{l}0 \\
6 \\
6\end{array}$ & $\frac{1}{2}$ & $\begin{array}{l}1.02 \\
=2\end{array}$ & $\begin{aligned} 4.01 \\
-1.09 \\
0.19 \\
0.19\end{aligned}$ & $\begin{array}{l}6 \\
6 \\
6 \\
6\end{array}$ & $\begin{array}{l}2 \\
2 \\
2 \\
2\end{array}$ & $\begin{array}{l}6 \\
6 \\
7 \\
8\end{array}$ & $\begin{array}{r}41.45 \\
4.58\end{array}$ & $\begin{array}{r}5.12 \\
-5.44 \\
-\quad 0.90\end{array}$ & $\begin{array}{l}8 \\
8 \\
8\end{array}$ & $\frac{3}{3}$ & $\begin{array}{l}3 \\
4 \\
5\end{array}$ & $\begin{array}{l}3.88 \\
=\end{array}$ & $\begin{array}{r}-3.86 \\
0.040 \\
0.85\end{array}$ \\
\hline $\begin{array}{l}4 \\
4 \\
4 \\
4\end{array}$ & $\begin{array}{l}6 \\
6 \\
6 \\
6\end{array}$ & $\begin{array}{l}3 \\
4 \\
5 \\
5\end{array}$ & $\begin{array}{l}6.50 \\
.5 \\
1.52\end{array}$ & $\begin{array}{r}0.050 \\
-7.01 \\
2.87 \\
2.98 \\
598\end{array}$ & $\begin{array}{l}6 \\
6 \\
6 \\
6\end{array}$ & $\begin{array}{l}\frac{2}{2} \\
\frac{2}{3} \\
3\end{array}$ & $\begin{array}{r}8 \\
10 \\
10\end{array}$ & $\begin{array}{l}4.48 \\
6.13 \\
7.213\end{array}$ & $\begin{array}{r}-4.00 \\
0.75 \\
-6.19 \\
7.07\end{array}$ & $\begin{array}{l}8 \\
8 \\
8 \\
8\end{array}$ & 年 & $\begin{array}{l}6 \\
7 \\
8 \\
9\end{array}$ & $\bar{\vdots}$ & $\begin{array}{r}-0.24 * \\
-0.24 \\
0.55 \\
0.55 \\
4.05\end{array}$ \\
\hline $\begin{array}{r}5 \\
5 \\
-5\end{array}$ & & 2 & $\begin{array}{r}5.16 \\
6.44\end{array}$ & $\begin{array}{r}4.50 \\
0.88 \\
6.34 \\
-3.34\end{array}$ & $\begin{array}{r}6 \\
-6 \\
6 \\
6\end{array}$ & 3 & $\frac{1}{2}$ & $\begin{array}{r}7.21 \\
10.89\end{array}$ & $\begin{array}{r}11.47 \\
1.30^{\circ} \\
\text {. }\end{array}$ & $\begin{array}{l}8 \\
9 \\
9\end{array}$ & & $\begin{array}{l}l \\
2 \\
4\end{array}$ & $\begin{array}{l}3.00 \\
1.65 \\
5.55\end{array}$ & $\begin{array}{r}4.05 \\
-7.76 \\
-7.31 \\
4\end{array}$ \\
\hline $\begin{array}{r}5 \\
-5 \\
5\end{array}$ & & $\begin{array}{l}4 \\
4 \\
6\end{array}$ & $\begin{array}{l}13.08 \\
13.55 \\
13.28\end{array}$ & $\begin{array}{r}12.22 \\
-822 \\
-10.22 \\
-10.75\end{array}$ & $\begin{array}{l}-6 \\
-6 \\
-6\end{array}$ & 3 & $\begin{array}{l}\frac{2}{3} \\
3\end{array}$ & $\begin{array}{l}6.20 \\
5.97\end{array}$ & $\begin{array}{r}-6.69 \\
5.12 \\
1.1,4\end{array}$ & $\begin{array}{l}9 \\
9 \\
9 \\
9\end{array}$ & . & $\begin{array}{r}6 \\
8 \\
10 \\
\end{array}$ & $\begin{array}{r}3.30 \\
5.56 \\
2.60 \\
2.60\end{array}$ & $\begin{array}{r}4.36 \\
-5.56 \\
2.20 \\
\end{array}$ \\
\hline $\begin{array}{r}-5 \\
5 \\
5 \\
5\end{array}$ & & $\begin{array}{r}6 \\
10 \\
10 \\
2\end{array}$ & $\begin{array}{l}13.35 \\
13.04\end{array}$ & $\begin{array}{r}-2.07^{*} \\
-11.52 \\
9.48 \\
0.36\end{array}$ & $\begin{array}{l}6 \\
6 \\
6 \\
6\end{array}$ & 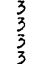 & $\begin{array}{l}4 \\
5 \\
6 \\
7 \\
7\end{array}$ & $\begin{array}{l}3.40 \\
3.24 \\
2.90\end{array}$ & $\begin{array}{r}3.38 \\
=0.990 \\
=3.13 \\
2.57\end{array}$ & $\begin{array}{l}9 \\
9 \\
9 \\
9 \\
9\end{array}$ & $\begin{array}{l}\frac{1}{1} \\
\frac{1}{1} \\
\frac{1}{1}\end{array}$ & $\begin{array}{l}2 \\
3 \\
4 \\
5\end{array}$ & $\begin{array}{l}5 \\
5.76\end{array}$ & $\begin{array}{l}=0.25^{\circ} 0^{\circ} \\
6.0 .93^{\circ} \\
-0.05^{\circ}\end{array}$ \\
\hline $\begin{array}{l}3 \\
5 \\
5\end{array}$ & $\frac{1}{1}$ & $\frac{1}{3}$ & - & $\begin{array}{r}0.30 \\
-1.04 \\
0.99 \\
\\
1.60\end{array}$ & $\begin{array}{l}6 \\
6 \\
6\end{array}$ & & $\begin{array}{r}8 \\
9 \\
10\end{array}$ & $\begin{array}{l}5.97 \\
1.67\end{array}$ & $\begin{array}{r}5.02 \\
-5.02 \\
-5.46 \\
1.60\end{array}$ & $\begin{array}{l}3 \\
9 \\
9 \\
9\end{array}$ & $\begin{array}{l}1 \\
1 \\
1\end{array}$ & $\begin{array}{l}6 \\
? \\
8\end{array}$ & $\begin{array}{l}4.04 \\
3.54 \\
3.500\end{array}$ & $\begin{array}{l}-0.03 \\
-3.35 \\
-3.55 \\
-3.47\end{array}$ \\
\hline $\begin{array}{l}5 \\
-5 \\
-5\end{array}$ & $\begin{array}{l}\frac{1}{1} \\
\frac{1}{1}\end{array}$ & $\begin{array}{l}2 \\
2 \\
\frac{2}{3}\end{array}$ & $\begin{array}{l}1.48 \\
4.35 \\
5.83\end{array}$ & $\begin{array}{r}1: 80^{\circ} \\
-3.70^{\circ} \\
-5.90\end{array}$ & $\begin{array}{r}6 \\
6 \\
-6\end{array}$ & $\begin{array}{l}4 \\
4 \\
4\end{array}$ & $\frac{1}{1}$ & $\begin{array}{l}\frac{1.65}{4.65} \\
2.76\end{array}$ & $\begin{array}{r}4.00 \\
-0.46^{*} \\
-0.96\end{array}$ & $\begin{array}{l}\text { g. } \\
\text { gे }\end{array}$ & $\begin{array}{l}\frac{1}{2} \\
\frac{1}{2} \\
\frac{2}{2}\end{array}$ & $\begin{array}{l}9 \\
9 \\
5 \\
5\end{array}$ & $\begin{array}{l}1.72 \\
1: 72 \\
=\end{array}$ & $\begin{array}{r}2.119 \\
1.30 \\
0.040\end{array}$ \\
\hline $\begin{array}{l}-5 \\
-5 \\
-5\end{array}$ & $\frac{1}{1}$ & $\begin{array}{l}3 \\
3 \\
4\end{array}$ & $\begin{array}{r}- \\
11.80 \\
4.08\end{array}$ & $\begin{array}{r}-1 \\
13.40^{\circ} \\
13.23 \\
4.58\end{array}$ & $\begin{array}{r}6 \\
-6 \\
-6\end{array}$ & 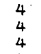 & $\begin{array}{l}2 \\
\frac{2}{3} \\
3\end{array}$ & $\begin{array}{l}3.17 \\
3.67\end{array}$ & $\begin{array}{l}=2.24 .4 \\
=3.15 \\
=3.09\end{array}$ & $\begin{array}{l}9 \\
\dot{g} \\
g\end{array}$ & $\begin{array}{l}2 \\
\frac{2}{2} \\
2\end{array}$ & $\begin{array}{l}6 \\
? \\
8\end{array}$ & $\begin{array}{l}4.99 \\
5.06\end{array}$ & $\begin{array}{r}5.69 \\
-5.39 \\
-5.19\end{array}$ \\
\hline $\begin{array}{l}5 \\
-5\end{array}$ & $\frac{1}{1}$ & $\begin{array}{l}5 \\
5\end{array}$ & 2.39 & $\begin{array}{l}1.01^{\circ} \\
2.82\end{array}$ & $\begin{array}{l}6 \\
6 \\
6\end{array}$ & 4 & $\begin{array}{l}4 \\
5 \\
6\end{array}$ & 5,66 & & & & & & \\
\hline & $\frac{1}{1}$ & $\begin{array}{l}6 \\
6 \\
7 \\
7\end{array}$ & $\begin{array}{l}3.94 \\
7.79\end{array}$ & $\begin{array}{r}0.19^{\circ} \\
-5.93^{\circ} \\
8.99\end{array}$ & $\begin{array}{l}6 \\
6 \\
6\end{array}$ & $\begin{array}{l}4 \\
4\end{array}$ & $\begin{array}{l}0 \\
? \\
8\end{array}$ & $\begin{array}{l}5.66 \\
\frac{6}{6}: 00 \\
3.03\end{array}$ & $\begin{array}{r}5.62 \\
-5.86 \\
-3.20\end{array}$ & & & & & \\
\hline & & $\begin{array}{l}8 \\
9 \\
9\end{array}$ & $\begin{array}{l}2.99 \\
3.929 \\
3.524\end{array}$ & $\begin{aligned}-2.53 \\
=4.53 \\
=4.35\end{aligned}$ & $\begin{array}{l}6 \\
? \\
7\end{array}$ & 4 & 9 & $\begin{array}{l}1.95 \\
3.24 \\
-2.24\end{array}$ & $\begin{array}{l}=1.72 \\
=\frac{1}{3} .00 \\
=1.66\end{array}$ & & & & & \\
\hline $\begin{array}{l}5 \\
5 \\
5\end{array}$ & 1 & $\begin{array}{l}\frac{11}{11} \\
12\end{array}$ & $\begin{array}{l}3.31 \\
2.06\end{array}$ & $\begin{array}{l}=3.55 .06 \\
=1.06 \\
1.88\end{array}$ & $\begin{array}{c}-7 \\
7\end{array}$ & & $\frac{1}{2}$ & 3.00 & $\begin{array}{r}-1.66 \\
-3.09 \\
0.56 .\end{array}$ & & & & & \\
\hline $\begin{array}{r}5 \\
-5 \\
-5\end{array}$ & $\begin{array}{l}2 \\
2 \\
2 \\
2\end{array}$ & $\frac{1}{1}$ & $\begin{array}{l}\frac{16.51}{10.38} \\
15.03\end{array}$ & $\begin{array}{r}-14.38 \\
-9.89 \\
-14.28\end{array}$ & $\begin{array}{l}? \\
? \\
?\end{array}$ & & $\begin{array}{r}6 \\
8 \\
10\end{array}$ & $\begin{array}{r}3.71 \\
10.31 \\
8.63\end{array}$ & $\begin{array}{r}2.36 \\
8.88 \\
-7.50\end{array}$ & & & & & \\
\hline $\begin{array}{l}5 \\
-5 \\
5\end{array}$ & $\begin{array}{l}2 \\
2 \\
2 \\
2\end{array}$ & $\begin{array}{l}2 \\
2 \\
3 \\
3\end{array}$ & $\begin{array}{r}7.99 \\
3.64 \\
3.06\end{array}$ & $\begin{array}{r}8.06 \\
-3.98 \\
-1.62\end{array}$ & $\begin{array}{l}7 \\
? \\
?\end{array}$ & & & 3.84 & $\begin{array}{l}-2.990 \\
-0.500 \\
-5.000\end{array}$ & & & & & \\
\hline $\begin{array}{c}-5 \\
5\end{array}$ & $\begin{array}{l}2 \\
2 \\
2\end{array}$ & $\begin{array}{l}3 \\
3 \\
4 \\
4\end{array}$ & $\begin{array}{l}2.39 \\
4.11 \\
5.11\end{array}$ & & $\begin{array}{c}-7 \\
? \\
0\end{array}$ & & $\frac{1}{2}$ & $\begin{array}{r}5.33 \\
2.76\end{array}$ & $\begin{array}{l}6.57 \\
2.87 \\
0.58\end{array}$ & & & & & \\
\hline 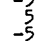 & & $\begin{array}{l}4 \\
5 \\
5\end{array}$ & $\begin{array}{l}.022 \\
1.92\end{array}$ & & $\begin{array}{c}-1 \\
-7 \\
-2\end{array}$ & & $\frac{3}{3}$ & 3.30 & $\begin{array}{r}0.70 \\
-3.72 \\
-1.232 .\end{array}$ & & & & & \\
\hline & & $\begin{array}{l}? \\
6 \\
? \\
8\end{array}$ & 5.92 & $\begin{array}{rl}-0.45 & -45^{\circ} \\
5 & 5.12\end{array}$ & $\begin{array}{c}7 \\
? \\
? \\
?\end{array}$ & & $\begin{array}{l}4 \\
5 \\
6\end{array}$ & 11.16 & $\begin{array}{r}-12.19 \\
-\frac{12}{1.39} \\
1.39\end{array}$ & & & & & \\
\hline & & $\begin{array}{l}80 \\
10\end{array}$ & $\begin{array}{l}7.41 \\
5.06\end{array}$ & $\begin{array}{r}7.20 \\
-\begin{array}{l}2.88 \\
0.48\end{array}\end{array}$ & $\begin{array}{l}7 \\
? \\
?\end{array}$ & & $\begin{array}{l}6 \\
? \\
8\end{array}$ & $\begin{array}{l}3.34 \\
3.448 \\
4.488\end{array}$ & $\begin{array}{r}4.43 \\
-8.22 \\
5 \\
5.05\end{array}$ & & & & & \\
\hline & & $\frac{11}{12}$ & .7.92 & & $\begin{array}{l}? \\
? \\
?\end{array}$ & & $\begin{array}{r}9 \\
10\end{array}$ & $\begin{array}{l}3.24 \\
1.96\end{array}$ & $\begin{array}{rl}3 & 3.03 \\
-2.25 & 23 \\
0.55\end{array}$ & & & & & \\
\hline - & & $\frac{1}{2}$ & $\begin{array}{r}4.58 \\
4.08 \\
4.08\end{array}$ & & $\begin{array}{l}7 \\
?\end{array}$ & & 1 & 5.93 & $\begin{array}{l}2.49 \\
6.49 \\
6.00\end{array}$ & & & & & \\
\hline $\begin{array}{l}-7 \\
-5 \\
-5\end{array}$ & & $\frac{2}{3}$ & $\begin{array}{l}4.68 \\
6.54 \\
2.59\end{array}$ & $\begin{array}{r}-4.50 \\
6.72 \\
2.20\end{array}$ & $\begin{array}{r}-6 \\
-7 \\
-7\end{array}$ & & $\begin{array}{l}\frac{1}{2} \\
\frac{2}{2}\end{array}$ & $=$ & $\begin{array}{r}8.00 \\
-0.54 \\
-0.21 \\
0.21\end{array}$ & & & & & \\
\hline - & & & & & $?$ & & 5 & & $\begin{array}{r}3.23 \\
-0.90 \\
-0.47\end{array}$ & & & & & \\
\hline 5 & & $\begin{array}{l}6 \\
6 \\
7 \\
8\end{array}$ & $\begin{array}{l}11.32 \\
6.40 \\
6.40\end{array}$ & $\begin{array}{r}-13.00 \\
-11.05 \\
6.34 \\
890\end{array}$ & $\begin{array}{l}? \\
? \\
? \\
?\end{array}$ & $\begin{array}{l}2 \\
2 \\
2 \\
2 \\
2\end{array}$ & $\begin{array}{l}? \\
? \\
7 \\
8\end{array}$ & $\begin{array}{l}6.81 \\
3.03 \\
2.09\end{array}$ & $\begin{array}{r}6.667 \\
1.76 \\
2.35\end{array}$ & & & & & \\
\hline 5 & & $\begin{array}{r}99 \\
10 \\
11\end{array}$ & $\begin{array}{l}5.07 \\
-08\end{array}$ & $\begin{array}{l}=5.63 \\
=5.52 \\
1.50\end{array}$ & $\begin{array}{l}? \\
? \\
? \\
0\end{array}$ & 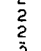 & $\begin{array}{r}9 \\
10\end{array}$ & $\begin{array}{l}5.70 \\
4=75\end{array}$ & $\begin{array}{r}-5.18 \\
-1.74 \\
5.72\end{array}$ & & & & & \\
\hline & & & $\begin{array}{l}1.58 \\
9.37 \\
9.32\end{array}$ & -0.89 & $\begin{array}{l}1 \\
?\end{array}$ & $\sum_{3}^{2}$ & 1 & $\begin{array}{l}4.75 \\
6.64\end{array}$ & $\begin{array}{r}5.72 \\
-6.12 \\
1.25\end{array}$ & & & & & \\
\hline & & $\frac{1}{2}$ & & & $?$ & $\frac{3}{3}$ & $\frac{2}{3}$ & $\begin{array}{l}4.95 \\
2.056\end{array}$ & $\begin{array}{r}4.755 \\
-2.75 \\
2.35\end{array}$ & & & & & \\
\hline $\begin{array}{l}-3 \\
-5 \\
-5\end{array}$ & & $\frac{2}{3}$ & $\begin{array}{l}4.55 \\
5.39\end{array}$ & $\begin{array}{l}4.84 \\
5.48 \\
1.88 .\end{array}$ & $\begin{array}{l}? \\
?\end{array}$ & 3 & $\begin{array}{l}4 \\
5 \\
6\end{array}$ & 10.25 & $\begin{array}{r}-0.25 \\
9.28 \\
6.12 \\
6.12\end{array}$ & & & & & \\
\hline & & $\begin{array}{l}4 \\
5 \\
6\end{array}$ & $\begin{array}{l}3.17 \\
4.08\end{array}$ & $\begin{array}{r}2.67 \\
-3.96 \\
-1.11 .\end{array}$ & $\begin{array}{l}? \\
? \\
?\end{array}$ & 3 & $\begin{array}{l}7 \\
8 \\
9\end{array}$ & $\begin{array}{l}0.94 \\
6.44 \\
1.84\end{array}$ & $\begin{array}{l}-2.41 \\
-6.24 \\
1.72\end{array}$ & & & & & \\
\hline$\stackrel{5}{5}$ & & $\begin{array}{l}6 \\
? \\
8\end{array}$ & 3.44 & $\begin{aligned} &=1.99^{\circ} \\
& 2.808\end{aligned}$ & $\begin{array}{l}? \\
? \\
?\end{array}$ & $\begin{array}{l}3 \\
3 \\
4\end{array}$ & $\begin{array}{r}99 \\
10 \\
\frac{1}{2}\end{array}$ & $\begin{array}{l}\frac{1}{2} .78 \\
2.83 \\
2.43\end{array}$ & $\begin{array}{r}1.41 \\
-2.48 \\
-2.36 \\
2.30\end{array}$ & & & & & \\
\hline & $\begin{array}{l}4 \\
4 \\
\end{array}$ & 109 & $\begin{array}{r}1.96 \\
9.07\end{array}$ & $\begin{array}{l}=2.63 \\
=0.03 \\
0.19\end{array}$ & $\begin{array}{l}? \\
? \\
? \\
?\end{array}$ & $\begin{array}{l}4 \\
4 \\
4\end{array}$ & $\begin{array}{l}\frac{2}{3} \\
4 \\
5\end{array}$ & $\begin{array}{l}5.27 \\
3.54\end{array}$ & $\begin{array}{r}=3.66 \\
=3.66 \\
-3.18\end{array}$ & & & & & \\
\hline-6 & & $\begin{array}{l}\frac{1}{2} \\
4 \\
4 \\
4\end{array}$ & $\begin{array}{r}5.43 \\
13.53 \\
13.65\end{array}$ & $\begin{array}{l}12.36 \\
-12.36\end{array}$ & $\begin{array}{l}7 \\
?\end{array}$ & 4 & $\begin{array}{l}? \\
6 \\
7\end{array}$ & $\begin{array}{l}2.00 \\
5.53 \\
5.59\end{array}$ & & & & & & \\
\hline
\end{tabular}


Table 2. Fractional atomic coordinates, and thermal parameters $\left(\AA^{2}\right)$, with estimated standard deviations in parentheses

$\begin{array}{lcccc}\text { Atom } & x & y & z & B \\ \mathrm{~N}(1) & 0.78075 & -0.16686 & 0.58746 & 1.98 \\ & (0.00041) & (0.00061) & (0.00027) & (0.08) \\ \mathrm{C}(2) & 0.84317 & -0.28202 & 0.67900 & 2.01 \\ & (0.00048) & (0.00076) & (0.00033) & (0.10) \\ \mathrm{N}(3) & 0.79256 & -0.15314 & 0.80836 & 1.82 \\ & (0.00043) & (0.00063) & (0.00029) & (0.08) \\ \mathrm{C}(4) & 0.69066 & 0.05258 & 0.79868 & 1.87 \\ & (0.00051) & (0.00071) & (0.00036) & (0.09) \\ \mathrm{C}(5) & 0.68238 & 0.04574 & 0.66323 & 1.99 \\ & (0.00050) & (0.00074) & (0.00034) & (0.09) \\ \mathrm{H}(1) & 0.817 & -0.206 & 0.472 & 2.14 \\ \mathrm{H}(2) & 0.914 & -0.458 & 0.628 & 2.14 \\ \mathrm{H}(4) & 0.622 & 0.150 & 0.892 & 2.14 \\ \mathrm{H}(5) & 0.619 & 0.153 & 0.611 & 2.14\end{array}$

Table 3. Bond lengths and angles

\begin{tabular}{llll}
$\mathrm{N}(1)-\mathrm{C}(2)$ & $1.349 \AA$ & $\mathrm{N}(1)-\mathrm{C}(2)-\mathrm{N}(3)$ & $111.3^{\circ}$ \\
$\mathrm{C}(2)-\mathrm{N}(3)$ & 1.326 & $\mathrm{C}(2)-\mathrm{N}(3)-\mathrm{C}(4)$ & $105 \cdot 4$ \\
$\mathrm{~N}(3)-\mathrm{C}(4)$ & 1.378 & $\mathrm{~N}(3)-\mathrm{C}(4)-\mathrm{C}(5)$ & 109.8 \\
$\mathrm{C}(4)-\mathrm{C}(5)$ & 1.358 & $\mathrm{C}(4)-\mathrm{C}(5)-\mathrm{N}(1)$ & $106 \cdot 3$ \\
$\mathrm{C}(5)-\mathrm{N}(1)$ & 1.369 & $\mathrm{C}(5)-\mathrm{N}(1)-\mathrm{C}(2)$ & 107.2 \\
$\mathrm{~N}(1)-\mathrm{H}(1)$ & 1.048 & $\mathrm{H}(1)-\mathrm{N}(1)-\mathrm{C}(2)$ & $129 \cdot 1$ \\
& & $\mathrm{H}(1)-\mathrm{N}(1)-\mathrm{C}(5)$ & 122.9 \\
$\mathrm{C}(2)-\mathrm{H}(2)$ & \multirow{2}{*}{1.082} & $\mathrm{H}(2)-\mathrm{C}(2)-\mathrm{N}(3)$ & 138.2 \\
& & $\mathrm{H}(2)-\mathrm{C}(2)-\mathrm{N}(1)$ & 110.4 \\
$\mathrm{C}(4)-\mathrm{H}(4)$ & \multirow{2}{*}{0.958} & $\mathrm{H}(4)-\mathrm{C}(4)-\mathrm{N}(3)$ & $115 \cdot 8$ \\
& & $\mathrm{H}(4)-\mathrm{C}(4)-\mathrm{C}(5)$ & 133.2 \\
$\mathrm{C}(5)-\mathrm{H}(5)$ & \multirow{2}{*}{1.031} & $\mathrm{H}(5)-\mathrm{C}(5)-\mathrm{C}(4)$ & 136.3 \\
& & $\mathrm{H}(5)-\mathrm{C}(5)-\mathrm{N}(1)$ & 117.4
\end{tabular}

Table 4. Distances from the least-squares plane and equation of the plane in $\AA$

$\begin{array}{cc}\text { Atom } & \text { Distance from plane } \\ \mathrm{N}(1) & 0.004 \AA \\ \mathrm{C}(2) & -0.004 \\ \mathrm{~N}(3) & 0.003 \\ \mathrm{C}(4) & -0.001 \\ \mathrm{C}(5) & -0.002 \\ 0.7314 x+0.5038 y-0.0460 z=3.5993\end{array}$

Table 5. Intermolecular distances

$$
\mathrm{N} \cdots \mathrm{N} \text { hydrogen bond }
$$

$\begin{array}{ccc}\text { Atom } i & \text { Atom } j & D(i j) \\ \mathrm{N}\left(1^{\prime}\right) & \mathrm{N}\left(3^{\prime \prime}\right) & 2 \cdot 86 \AA\end{array}$

Van der Waals distances less than $4.0 \AA$ between atoms. The symmetry relationship of the atoms concerned is denoted by superscripts as follows:

$\begin{array}{lrrr}\prime & x & y & z \\ \prime \prime & x & -\frac{1}{2}-y & -\frac{1}{2}+z \\ \prime \prime & 1-x & -\frac{1}{2}+y & \frac{3}{2}-z \\ \text { iv } & x & -1+y & z \\ \text { v } & x & -\frac{1}{2}-y & \frac{1}{2}+z \\ \text { vi } & 1-x & \frac{1}{2}+y & 3-z\end{array}$

Table 5 (cont.)

$\begin{array}{lll}\text { Atom } i & \text { Atom } j & D(i j) \\ \mathrm{N}\left(1^{\prime}\right) & \mathrm{C}\left(2^{\prime \prime}\right) & 3 \cdot 80 \AA \\ \mathrm{N}\left(1^{\prime}\right) & \mathrm{C}\left(4^{\prime \prime}\right) & 3 \cdot 82 \\ \mathrm{~N}\left(1^{\prime}\right) & \mathrm{C}\left(4^{\prime \prime \prime}\right) & 3 \cdot 53 \\ \mathrm{~N}\left(1^{\prime}\right) & \mathrm{C}\left(5^{\prime \prime \prime}\right) & 3.52 \\ \mathrm{C}\left(2^{\prime}\right) & \mathrm{N}\left(3^{\prime \prime}\right) & 3.85 \\ \mathrm{C}\left(2^{\prime}\right) & \mathrm{C}\left(4^{\text {iv }}\right) & 3.76 \\ \mathrm{C}\left(2^{\prime}\right) & \mathrm{C}\left(5^{\text {iv }}\right) & 3.84 \\ \mathrm{C}\left(2^{\prime}\right) & \mathrm{C}\left(5^{\prime \prime \prime}\right) & 3.63 \\ \mathrm{~N}\left(3^{\prime}\right) & \mathrm{C}\left(5^{\mathrm{v}}\right) & 3.79 \\ \mathrm{~N}\left(3^{\prime}\right) & \mathrm{C}\left(5^{\prime \prime \prime}\right) & 3.83 \\ \mathrm{C}\left(4^{\prime}\right) & \mathrm{C}\left(5^{\text {vi }}\right) & 3.76 \\ \mathrm{C}\left(4^{\prime}\right) & \mathrm{C}\left(5^{\prime \prime \prime}\right) & 3.81 \\ \mathrm{C}\left(5^{\prime}\right) & \mathrm{C}\left(5^{\text {vi }}\right) & 3.63 \\ \mathrm{C}\left(5^{\prime}\right) & \mathrm{C}\left(5^{\prime \prime \prime}\right) & 3.63\end{array}$

imidazole molecule, together with the distances of atoms from this plane, is given in Table 4. These structural data were obtained with programs written by $\mathrm{Chu}$ (1963) for the IBM 1620 computer.

\section{Discussion}

Bond lengths and angles of the imidazole molecule are shown in Fig. 4. As seen in Table 4, the imidazole ring is completely planar within the error limits. The positions of the hydrogen atoms are less certain; none of them lies more than $0 \cdot 16 \AA$ out of the plane of the ring. In analogy to other heterocyclic ring systems, imidazole may be represented as a resonance hybrid with contributions from the following formulae (see e.g. Hofmann, 1953):<smiles></smiles>

(I)<smiles></smiles>

(II)<smiles></smiles>

(III)

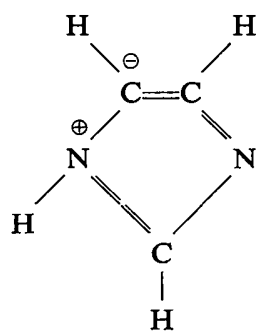

(IV)<smiles>[CH]1[CH][NH2+]C1</smiles>

(V)
An estimate was made of the contributions of the limiting structures (I) to (V) from the bond lengths in Table 2 in the following way. The double-bond character of the $\mathrm{C}-\mathrm{C}$ bond was obtained from the curve of bond length versus bond order given by Pauling 
(1960); for the $\mathrm{C}-\mathrm{N}$ bonds an analogous curve constructed by Wheatley (1954) was used. The percentages estimated were:

$\begin{array}{ll}\mathrm{N}(1)-\mathrm{C}(2) & 37 \% \\ \mathrm{C}(2)-\mathrm{N}(3) & 51 \\ \mathrm{~N}(3)-\mathrm{C}(4) & 24 \\ \mathrm{C}(4)-\mathrm{C}(5) & 68 \\ \mathrm{C}(5)-\mathrm{N}(1) & 27\end{array}$

The sum of the double-bond percentages is $207 \%$, which is as close to the expected $200 \%$ as could have been hoped for. By assuming the shortening of N(1)$\mathrm{C}(2)$ to be due only to formulae (II) and (IV), etc., we evaluated the contributions of (I) to (V) as $38 \%$ (I), $28 \%$ (II), $15 \%$ (III), $8 \%$ (IV) and $11 \%$ (V). Although we must not attach too much importance to these values, there seems to be no doubt that the non-polar form (I) is the most important, with (II) as a good second and minor contributions from the forms with a negative formal charge on carbon. The relatively large total contribution of the polar forms (II)-(V) is in agreement with the high dipole moment of imidazole. Form (II) especially with its positive charge on N(1) and negative charge on $\mathrm{N}(3)$ will contribute to the strength of the attraction between the pyridine nitrogen of one molecule with the imino nitrogen of the next molecule, generated by the glide plane $c$. In this way, strings of molecules along the $c$ axis are formed, as seen in projection along the $a$ and $b$ axes in Figs. 5 and 6 respectively. Even in non-polar solvents strings of several molecules appear to persist (Hofmann, 1953). In this connection, it is interesting to note the strong anisotropy of the thermal expansion. Since the axial angle $\beta$ changes, and in some directions the expansion coefficient is negative, it is impossible to calculate the expansion ellipsoid (Wooster, 1938, p.26) from the change in $d$ values. Table 6 shows some values for the expansion coefficient in various directions.

The slightly negative thermal expansion along the $c$ axis might be due to a slight change in orientation of the molecules at room temperature. In this respect

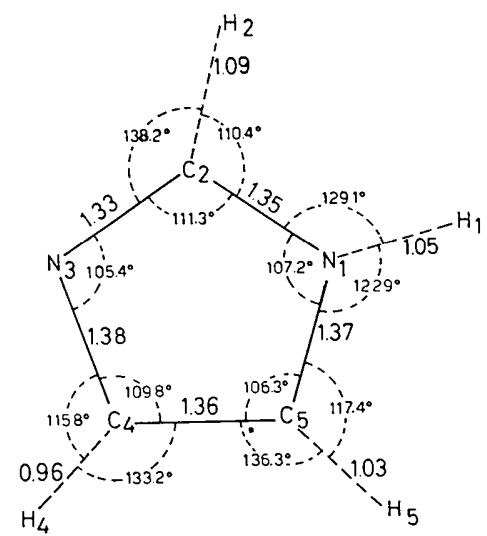

Fig.4. Bond lengths and angles in the imidazole molecule.

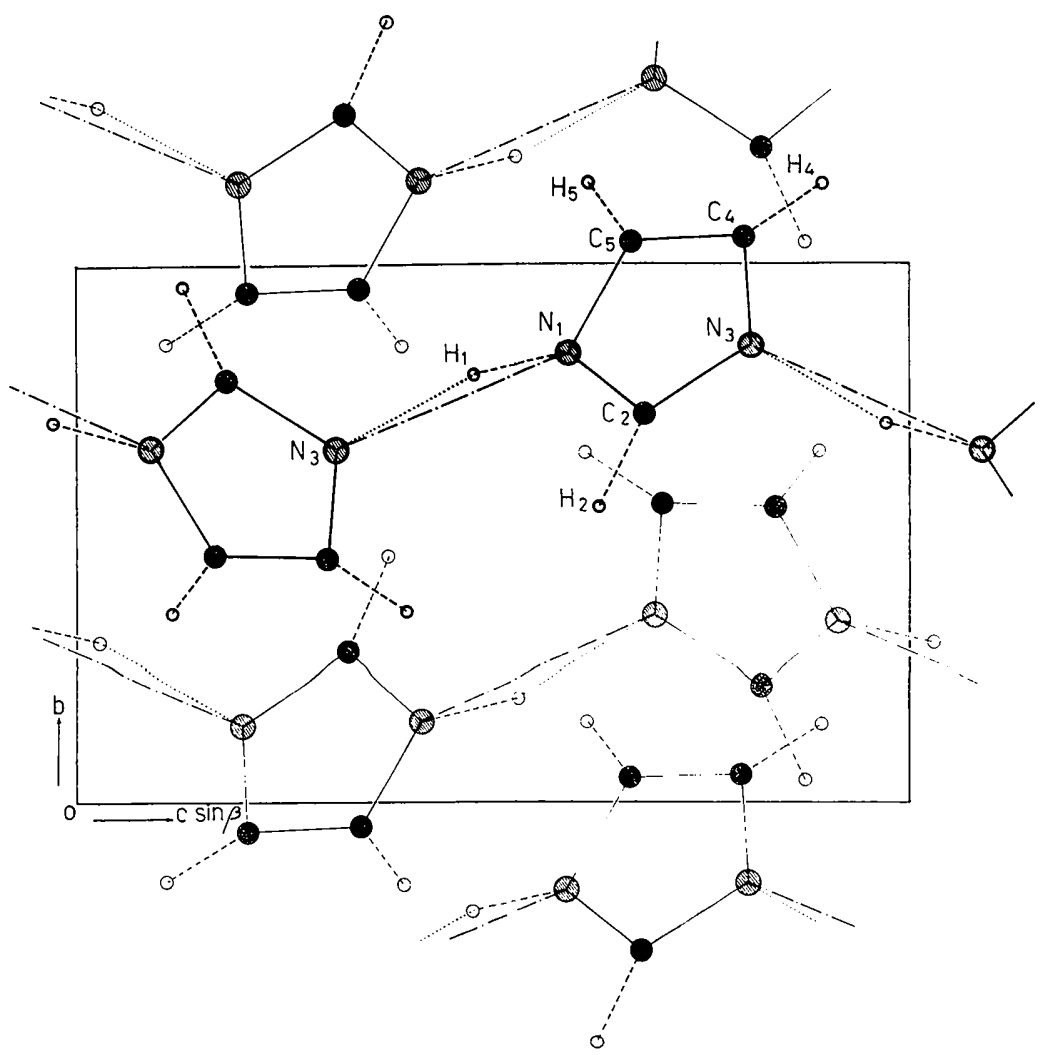

Fig. 5. Imidazole. Projection of the structure along the $a$ axis. 


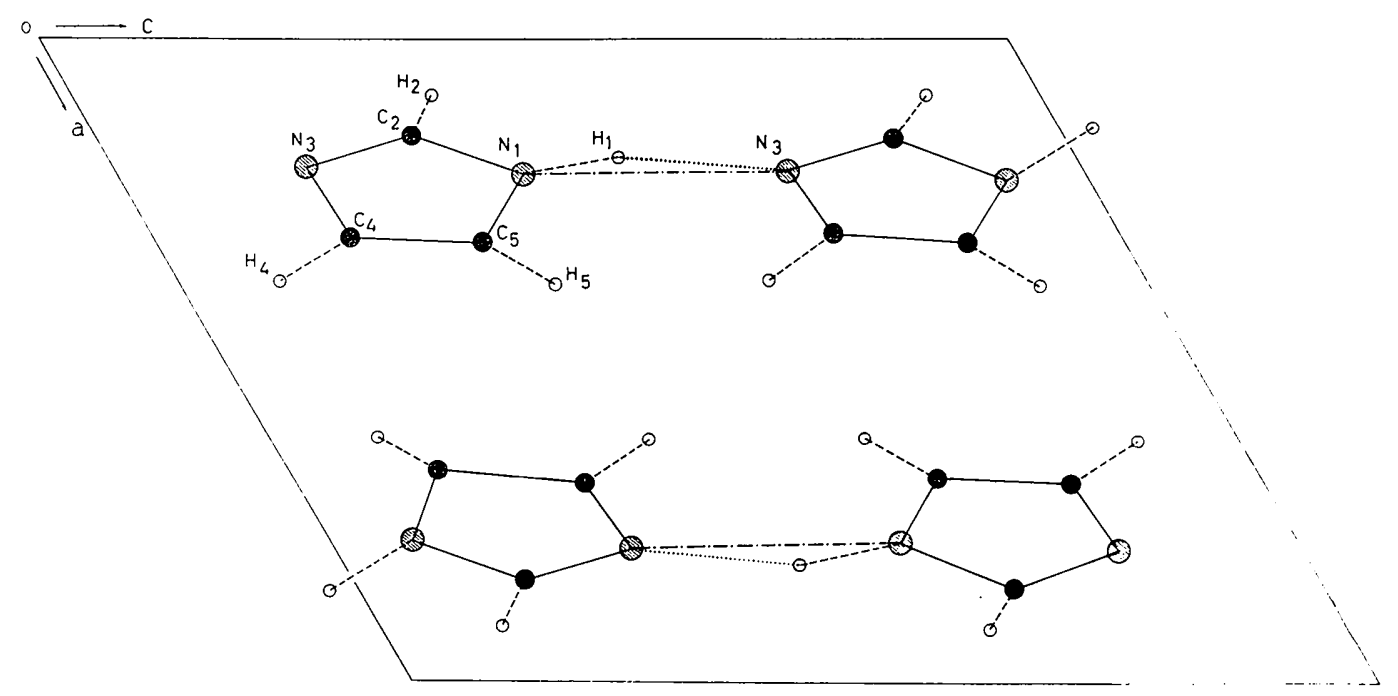

Fig. 6. Imidazole. Projection of the structure along the $b$ axis.

Table 6. Thermal expansion coefficient of imidazole in various directions

$\begin{array}{crcc}\text { Direction } & \alpha \times 10^{4} & \text { Direction } & \alpha \times 10^{4} \\ {[010]} & 0.90 & (100) & 2 \cdot 2 \\ {[100]} & 1.95 & (70 \overline{2}) & 2.3 \\ {[001]} & -0.14 & (60 \overline{4}) & 2 \cdot 3 \\ & & (001) & 0.83 \\ & & (7,0,10) & -0.15\end{array}$

the promised refinement of the room temperature data (Will, 1963) is awaited with interest.

The author wishes to express her gratitude to Prof. C. H. MacGillavry, Dr C.H.Stam and Mr A.Kreuger for their help during her stay in Amsterdam; to Prof. G.A. Jeffrey for allowing her to use the computation facilities and program of the Laboratory of Crystallography of the University of Pittsburgh.

\section{References}

CHU, S. (1963). Unpublished work.

Cochran, W. A. (1951). Acta Cryst. 4, 408.

García Blanco, S., \& Martinez-Carrera, S. (1958). An. Real. Soc. Fis. Quim. 54 (A), 75.

GreENwood, G. (1926). Mineral. Mag. 21, 1.

Hofmann, K. (1953). The Chemistry of Heterocyclic Compounds, Vol.I.

KReUGer, A. (1955). Acta Cryst. 8, 348.

Pauling, L. (1960). The Nature of the Chemical Bond. Ithaca: Cornell Univ. Press.

Phillips, D. C. (1956). Acta Cryst. 9, 819.

SHiono, R. (1962). Program No.376, I.U.Cr. World List of Crystallographic Computing Programs.

WALLWORK, S. C. (1962). Acta Cryst. 15, 758.

Wheatley, P. J. (1955). Acta Cryst. 8, 224.

WILL, G. (1963). Z. Kristallogr. 119, 1.

Wooster, W. A. (1938). A Text-book on Crystal Physics. Cambridge Univ. Press. 